\title{
Urdu translation and validation of premature ejaculation diagnostic tool (PEDT)
}

\author{
Muhibullah Bangash ${ }^{1}$, Wajahat Aziz², \\ Mohammad Shoaib ${ }^{3}$, M Hammad Ather
}

\begin{abstract}
Objective: To validate an Urdu translation of premature ejaculation diagnostic tool (PEDT) by analyzing the association of this diagnostic tool with the clinical diagnosis of premature ejaculation (PE) and intravaginal ejaculatory latency time (IELT).

Methods: This cross-sectional study was conducted at the urology section of the Aga Khan University Hospital, Karachi, for six months duration, from July 2018 to December 2018. In our study 108 subjects, aged 20 to 50 years, who were in a stable sexual relationship (heterosexual) for a minimum duration of six months, were asked to fill the Urdu version of PEDT, 61 with PE and 47 without PE.

Results: The two groups matched for mean age, duration of relationship and education level. The duration of $1.2( \pm 0.5)$ minutes was the mean self-estimated IELT in the PE group and $3.7 \pm 0.9$ minutes in patients without PE. There was a significant negative correlation of 0.6 ( $p$-value $<0.001)$ between the PEDT score and self-estimated IELT. The test-retest reliability for each item was found to be significant for each individual item $(\geq 0.84, p$-value $<0.001)$ and 0.94 was the correlation coefficients of the total score, showing an excellent test-retest reliability. 0.93 was the Cronbach's alpha score ( $95 \%$ Confidence interval $=0.905-0.948$ ) indicating a significant internal consistency in the Urdu version of PEDT.

Conclusions: The Urdu version of PEDT is a valid tool to define and quantify PE objectively, with adequate internal consistency. This version of PEDT has a good negative correlation with self-estimated IELT and excellent correlation with clinical PE.
\end{abstract}

KEYWORDS: Premature ejaculation (PE), Premature Ejaculation Diagnostic Tool (PEDT), Intra-vaginal ejaculatory latency time (IELT).

doi: https://doi.org/10.12669/pjms.36.6.2405

How to cite this:

Bangash M, Aziz W, Shoaib M, Ather MH. Urdu translation and validation of premature ejaculation diagnostic tool (PEDT). Pak J Med Sci. 2020;36(6):1241-1245. doi: https://doi.org/10.12669/pjms.36.6.2405

This is an Open Access article distributed under the terms of the Creative Commons Attribution License (http://creativecommons.org/licenses/by/3.0), which permits unrestricted use, distribution, and reproduction in any medium, provided the original work is properly cited.

1. Muhibullah Bangash, MBBS.

Urology Resident. (Year-VI),

2. Wajahat Aziz, MBBS, FCPS (Urol),

Senior Instructor,

3. Mohammad Shoaib, MBBS.

Urology Resident. (Year-V),

4. M Hammad Ather, MBBS, FCPS (Urol), FRCS, FEBU.

Prof and Section Head of Urology,

1-4: Urology Section, Aga Khan University Hospital, Karachi, Pakistan.

Correspondence:

Muhibullah Bangash, MBBS, Urology Resident (Year-VI), Department of Surgery, Aga Khan University Hospital, PO Box 3500, Stadium Road, Karachi, 74800, Pakistan. E-mail: muhibbangash@gmail.com

* Received for Publication:

* Revision Received:

* Corrected and Edited:

* Accepted for Publication:
February 22, 2020

March 28, 2020

July 14, 2020

July 18, 2020

\section{INTRODUCTION}

Premature ejaculation (PE) is an ejaculatory disorder with a highly variable estimated prevalence which is partly explained by the lack of standardized definitions and diagnostic tools. ${ }^{1}$ There are various definitions of premature ejaculation in literature. In the Diagnostic and Statistical Manual of Mental Disorders-IV-Text Revision (DSM-IV-TR), PE was defined according to patient description and clinician assessment of a few other factors. ${ }^{2}$ The International Society for Sexual Medicine (ISSM) adopted the first evidencebased objective definition of PE. ${ }^{3}$ Premature ejaculation (lifelong and acquired) is a male sexual dysfunction with following features: 
1. Ejaculation that always occurs before or within about one minute of vaginal penetration (lifelong PE) or a clinically reduced in latency time, to about three minutes or less (acquired PE).

2. Unable to delay ejaculation on all or nearly allvaginal penetrations.

3. Negative personal consequences, such as distress, bother, frustration, and/or the avoidance of sexual intimacy.

Several questionnaires were developed to objectively diagnose premature ejaculation. Two of the most commonly used tools to diagnose PE, based upon patient-reported outcomes are Arabic Index of Premature Ejaculation (AIPE) and Premature Ejaculation Diagnostic Tool (PEDT). PEDT has been the most widely used tool in many countries and it is a brief questionnaire comprising of five items. ${ }^{4}$ PEDT score of more than 11 indicates PE diagnosis, a score of 9 or 10 implies a probable $\mathrm{PE}$, while a score of $<8$ indicates no PE. PEDT has standardized the diagnosis of PE and has already been translated and validated in various languages. However, an Urdu translation and validation of PEDT does not exist. Urdu is the language widely spoken and understood in Pakistan with a population of around 217 million (worldmeter.info). ${ }^{5}$

PE has a negative effect on quality of life, which extends beyond sexual dysfunction. Despite these serious psychological consequences of PE, only a few men seek treatment. Language is an important barrier both for patients and physicians involved in the management of PE. Patients are more comfortable in explaining their condition when they can express in their own language. Also, the availability of diagnostic tools in local languages allow for quantification and follow-up. We aimed to develop a validated translation of PEDT to be used in the Urdu speaking population. Urdu being a living language, spoken by almost a 100 million people around the world ${ }^{6}$ (ORGI, 2011).

\section{METHODS}

This Cross-Sectional study was conducted at the urology section of the Aga Khan University Hospital, Karachi, for six months duration i.e., from July 2018 to December 2018 after institutional and ethical review committee approval (ERC Number: 5373-Sur-ERC-18, Dated: $6^{\text {th }}$ June, 2018). The translation was performed using a multistep process described by WHO. ${ }^{7}$ PEDT (original
English version) was translated into Urdu by two experienced urologists (native URDU speaking), individually, with more than five years of clinical experience to diagnose and treat men with sexual dysfunction, including PE. Combining the two translations, a single Urdu version of PEDT was developed, which was further discussed with the panel of experts and modified as suggested. This initial Urdu version was then translated into English by a bilingual linguist and the final Urdu version of PEDT was developed, by comparing it with the original English PEDT version.

All patients with age 20 to 50 years, who were in a stable sexual relationship (heterosexual) for a minimum duration of six months, visiting the urology clinic, that agreed to participate in the study were included. Study participants were given information about the study procedure and informed written consent was obtained. Study participants filled the PEDT (Urdu) before and after consultation. A pilot study was conducted before the actual validation, including 20 participants (10 with PE and 10 without PE), using process described by $\mathrm{WHO}^{11}$. This version was accurate in diagnosing PE and the questions were easy to read and understand by the participants. Data was collected for demographics including age, comorbid conditions, frequency of intercourse, marital status, duration of the relationship, education level, self-reported IELT, type of PE (Lifelong vs acquired). Patients with concomitant erectile dysfunction, on psychiatric medications, major diseases (angina, heart failure, kidney or liver disease, and psychological disorders) and patients with abnormal penile shape were excluded from the study.

SPSS $^{\text {TM }}$ v21.0 was used for analyzing the data. Internal consistency, test-retest reliability, and validity was checked by performing psychometric analysis. Cronbach's alpha $\geq 0.70$ was considered to be a significant level of internal consistency.

- Self-estimated IELT and PEDT correlation was evaluated with the Spearman correlation.

- Physician-diagnosed PE and PEDT correlation was evaluated with Spearman correlation.

- Test-retest reliability was evaluated with Pearson correlation by comparing PEDT scores before and after consultation and 0.70 was the minimum acceptable level.

\section{RESULTS}

Initially, 140 eligible participants filled all the questionnaires at the first interview. During the 
Urdu version of PEDT for PE evaluation

Table-I: Demographic profile of the entire cohort.

\begin{tabular}{lcccc}
\hline & Total $(n=108)$ & Clinical PE $(n=61)$ & Clinical No PE $(n=47)$ & $p$-value \\
\hline Age (years) & $34.9 \pm 7.84$ & $33.77 \pm 8.70$ & $34.96 \pm 6.15$ & 0.429 \\
Frequency of Intercourse (weekly) & $2.07 \pm 0.84$ & $1.95 \pm 0.87$ & $2.17 \pm 0.86$ & 0.215 \\
Duration of Relationship & $7.28 \pm 6.02$ & $7.34 \pm 6.73$ & $7.20 \pm 5.00$ & 0.904 \\
Education [n (\%)] & & & \\
- Primary school & $34(31.48)$ & $18(16.66)$ & $16(14.81)$ & 0.670 \\
- High school & $41(37.96)$ & $25(23.14)$ & $16(14.81)$ & \\
- Graduate & $33(30.55)$ & $17(15.74)$ & $16(14.81)$ & \\
Self-reported IELT (minutes) & $2.32 \pm 1.39$ & $1.18 \pm 0.52$ & $3.70 \pm 0.91$ & $<0.001$ \\
PEDT score Pre-consultation & $10.62 \pm 4.39$ & $13.39 \pm 2.66$ & $7.02 \pm 3.51$ & $<0.001$ \\
PEDT score Post-consultation & $10.29 \pm 4.39$ & $13.20 \pm 2.58$ & $6.53 \pm 3.23$ & $<0.001$ \\
\hline
\end{tabular}

second interview, 32 participants dropped out. In the final study, 61 participants having clinical PE and 47 men without PE were enrolled for validation. Among all patients having PE, 50\% $(n=31)$ had acquired PE. The mean ages of the patients with PE and without PE were 33.77 $( \pm 8.70)$ years and $34.96( \pm 6.15)$ years, respectively. Frequency of intercourse was approximately twice weekly in both the groups. Mean age, frequency of intercourse, duration of relationship and level of education were similar in both groups. Mean selfestimated IELT was lower in the PE patients, (1.2 \pm $0.52 \mathrm{~min})$ as compared to the non-PE patients (3.7 \pm $0.91 \mathrm{~min}$ ). (Table-I)

Regarding the diagnostic value of Urdu version of PEDT score, we found a negative correlation of 0.6 between the PEDT and self-estimated IELT ( $p$-value $<0.001)$. For PEDT score, using cutoff value of 8 ( $\mathrm{PE}>8$ and no-PE $\leq 8)$ there was a false positive rate of $12.8 \%$ (6 men) i.e. PEDT indicated $\mathrm{PE}$, whereas the experts suggested that men did not have clinical PE. There was a false negative rate of $11.5 \%$ (7 men) where PEDT score indicated

Table-II: Sensitivity and Specificity of PEDT scores of greater/lesser than 8 with clinical diagnosis of PE.

\begin{tabular}{lccc}
\hline & $\begin{array}{c}\text { Physician } \\
\text { Diagnosis } \\
\text { PE \% }(n)\end{array}$ & $\begin{array}{c}\text { Physician } \\
\text { Diagnosis } \\
\text { No PE \% }(n)\end{array}$ & Total \\
\hline PEDT $>8$ & $88.52 \%(54)$ & $12.76 \%(6)$ & 60 \\
PEDT 8 or less & $11.48 \%(7)$ & $87.23 \%(41)$ & 48 \\
\hline Total & $\mathrm{n}=61$ & $\mathrm{n}=47$ & \\
\hline
\end{tabular}

no-PE while the expert diagnosed as clinical PE. Sensitivity and specificity of the Urdu version of PEDT score were $88.5 \%$ (95\% Confidence interval $(\mathrm{CI})=77.78 \%-95.26 \%)$ and $87.23 \%(95 \% \mathrm{CI}=$ $74.26 \%-95.17 \%$ ) respectively (Table-II).

There was excellent internal consistency in Urdu version of PEDT, calculated as 0.93 Cronbach's alpha score $(95 \% \mathrm{CI}=0.905-0.948)$. The testretest reliability for each item was evaluated with Pearson correlation and found to be significant for all five items and 0.941 (P-value < 0.001) was the correlation coefficient of the total PEDT score (Table-III). Moreover, mean PEDT scores preconsultation and post-consultation were similar (13.4 \pm 2.66 and 13.2 \pm 2.58 respectively) in the PE group. Mean PEDT scores pre-consultation and post-consultation were also similar in the non-PE group (7.0 \pm 3.50 and $6.53 \pm 3.23$ respectively).

\section{DISCUSSON}

Premature ejaculation evaluation with PEDT is an excellent tool that helps clinicians in objectively defining and quantifying the condition. PE has a highly variable estimated prevalence worldwide and very limited literature is available from local studies performed on sexual dysfunction. Only few studies done in our country have addressed premature ejaculation. One of the study done in a tertiary care hospital in Abbottabad, reported $13 \%$ prevalence of premature ejaculation and it was reported to be the second most common sexual dysfunction. ${ }^{8}$ Other studies from tertiary care hospitals in Pakistan, reported a variable

Table-III: Test re-test reliability of each question and total PEDT score.

\begin{tabular}{lcccccc}
\hline & Question: 1 & Question: 2 & Question: 3 & Question: 4 & Question: 5 & Total \\
\hline $\mathrm{R}$ & 0.948 & 0.843 & 0.852 & 0.919 & 0.945 & 0.941 \\
$\mathrm{p}$-value & $<0.001$ & $<0.001$ & $<0.001$ & $<0.001$ & $<0.001$ & $<0.001$ \\
\hline
\end{tabular}

$\begin{array}{lllll}\text { Pak J Med Sci September - October } 2020 & \text { Vol. } 36 & \text { No. } 6 & \text { www.pjms.org.pk } 1243\end{array}$ 
estimated prevalence of PE i.e., 30-34\%, ${ }^{9} 21 \%$ ${ }^{10}$ and $24-27 \% .{ }^{11}$ Various characteristics of PEDT have been validated in other regional languages i.e., Korean, ${ }^{12}$ Persian, $^{13}$ Turkish $^{14}$ and Chinese. ${ }^{15}$ National language of Pakistan is Urdu and easily understood by most people in this region with a population of around 217 million, and a language understood by nearly 100 million. Previously Urdu version of International Index of Erectile Function (IIEF) ${ }^{16}$ and Female Sexual Function Index (FSFI) ${ }^{17}$ has been validated. This has helped in opening up discussion about sexuality among physicians and patients in the Urdu speaking population. In the initial phase of translation, there was some difficulty in finding easily comprehensible terms in Urdu language for English words used in the original tool. Due to social taboos surrounding sex, there was the scarcity of easily understandable, non-obscene words particularly related to sexual intimacy in Urdu. As it is a private interaction and couples are hesitant in sharing it frankly even among them so words like "ejaculation" and "penetration" are rarely used in formal conversation in Urdu. As PEDT is a self-administered questionnaire, easily comprehensible terms are important to use for a validated questionnaire. We found that patients were able to understand the translation probably due to the context in which it was used and mostly did not require any assistance for filling the proforma. Our results have shown that our translation is a reliable and simple tool for common Urdu speaking people.

Our study showed good internal consistency and excellent stability for the PEDT (Urdu version) on test-retest reliability test than most of the previous published translations in other languages. This may be consequent to the fact that the study was conducted in the urology clinic of a tertiary care private hospital with an active andrology service. In our study there was a strong relationship of the calculated PEDT score for the Urdu version with self-estimated IELT, both for patients with and without PE. This is consistent with the results of Persian and Chinese validation studies of this tool (Huang et al 2014). However, PE cannot be diagnosed with time alone, this is reflected in a relatively lower correlation of 0.6 between IELT and PEDT score. Moreover, there are other factors e.g. situational PE, intermittent symptoms, effect on relationship and self-confidence which need to be taken into account when making a diagnosis of
PE, this is shown by some false positive and false negative results when PEDT score was compared with a physician diagnosis of PE.

The five items in PEDT score are closely related, so our study showed good internal consistency of Urdu version of PEDT with Cronbach's alpha score of 0.93 similar to the study by Pakpour and colleague, for the Iranian version of PEDT, alpha $=$ 0.89. ${ }^{18}$ It is interesting to note that Urdu and Persian are closely related languages in terms of structure and even words used. Correlating the results of the Urdu version of the PEDT questionnaires filled twice, before and after the clinic visit assessed the test-retest reliability and strong correlation was observed. This is a potential limitation as there was a small interval between the two interviews. However, a longer interval between the two interviews can introduce bias in terms of change in symptoms and sometimes by use of treatment including psychosexual counseling by a physician. $^{19}$

Many studies have validated diagnostic tools in the Urdu language (e.g., Urdu translation of the Hamilton Rating Scale for Depression ${ }^{20}$ ), but none was available for the PEDT in the Urdu language. The strengths of the study are that it is the first to translate and validate PEDT in Urdu and WHO guidelines for translation and adaptation of instruments were followed. Although PE has a variable prevalence but it is underreported worldwide, as admitting having $\mathrm{PE}$ is still considered a taboo by many people in our country. There was no objective tool yet to define PE objectively in the Urdu language for doing research work specifically on PE. This would also help the researchers in comparing drugs for PE and will help clinicians to see the results of drugs administered for PE in Urdu speaking patients, by assessing improvement in the PEDT scores.

Limitations of the study: However, our study is limited in terms of generalizability of results as it is a single-center study conducted at a tertiary care hospital. Nevertheless, with the availability of this validated tool, we can now apply this tool for a wider Urdu speaking population. Our results suggest that the Urdu version of PEDT is a valid tool that can be reliably used to diagnose premature ejaculation objectively among the Urdu-speaking population. 


\section{CONCLUSION}

The Urdu translation of PEDT is a valid tool to objectively define PE among Urdu speaking patients easily. It showed adequate internal consistency. This version of PEDT has a good correlation with both self-estimated IELT and clinician diagnosis of PE. To establish it as a routine PE diagnosing tool for Urdu speaking patients, further population-based and multi-center studies are required.

\section{Grant Support \& Financial Disclosures: None.}

\section{REFERENCES}

1. Saitz, TR, Serefoglu EC. The epidemiology of premature ejaculation. Transl Androl Urol. 2016;5(4):p.409.

2. Cooper R, Diagnosing the diagnostic and statistical manual of mental disorders. fifth ed. 2018:p.443. Routledge. doi: 10.4324/9780429473678

3. Serefoglu EC, McMahon CG, Waldinger MD, Althof SE, Shindel A, Adaikan G, et al. An evidence-based unified definition of lifelong and acquired premature ejaculation: report of the second International Society for Sexual Medicine Ad Hoc Committee for the Definition of Premature Ejaculation. Sex Med. 2014;2(2):41-59. doi: $10.1002 / \mathrm{sm} 2.27$

4. Symonds T, Perelman MA, Althof S, Giuliano F, Martin M, May K, et al. Development and validation of a premature ejaculation diagnostic tool. Eur Urol. 2007;52(2):565-573.

5. Accessed on https://www.worldometers.info/worldpopulation/pakistan-population/ accessed on 29th Sept 2019.

6. ORGI. “Census of India: Comparative speaker's strength of Scheduled Languages-1951, 1961, 1971, 1981, 1991, 2001 and 2011" (PDF).

7. World Health Organization. Process of translation and adaptation of instruments. http://www. who. int/ substance_abuse/research_tools/translation/en/. 2009. assessed on 24 April, 2018.

8. Khawaja MY. Frequency of male sexual dysfunction in a tertiary care hospital. J Med Sci 2005;13(2):140-142.

9. Taj R, Mufti M, Khan A, Rehman G. A study of sexual dysfunction in depressed males. Ann Pak Inst Med Sci. 2005;1(1):37-39.

10. Shaikh IA, Kumar S, Ujjan ID, Shaikh S. Frequency of sexual dysfunctions in type 2 diabetic males. J Liaquat Uni Med Health Sci. 2010;9(3):148-150.

11. Ahmer S, Khan F, Bhimani M. Psychiatric presentations of sexual dysfunction in Pakistan. J Pak Psych Soc. 2010;7(1):18-22.
12. Kam SC, Han DH, Huh JH, Lee SW. Development and validation of a Korean version of the premature ejaculation diagnostic tool (PEDT). Korean J Androl. 2009;27(3):185-193.

13. Pakpour AH, Yekaninejad MS, Nikoobakht MR, Burri A, Fridlund B. Psychometric properties of the Iranian version of the premature ejaculation diagnostic tool. Sex Med. 2014;2(1):31-40.

14. Serefoglu EC, Cimen HI, Ozdemir AT, Symonds T, Berktas $\mathrm{M}$, Balbay MD. Turkish validation of the premature ejaculation diagnostic tool and its association with intravaginal ejaculatory latency time. Int J Impot Res. 2009;21(2):139-144

15. Huang YP, Chen B, Ping $\mathrm{P}$, Wang HX, Hu K, Zhang T, et al. The premature ejaculation diagnostic tool (PEDT) linguistic validity of the Chinese version. J Sex Med. 2014;11(9):2232-2238. doi: 10.1111/jsm.12612

16. Mahmood MA, Rehman KU, Khan MA, Sultan T. Translation, cross-cultural adaptation, and psychometric validation of the 5-item International Index of Erectile Function (IIEF-5) into Urdu. J Sex Med. 2012;9(7):18831886. doi: 10.1111/j.1743-6109.2012.02714.x

17. Rehman KU, Asif Mahmood M, Sheikh SS, Sultan $T$, Khan MA. The Female Sexual Function I ndex (FSFI): Translation, Validation, and Cross-Cultural Adaptation of an U rdu Version "FSFI-U”. Sex Med. 2015;3(4):244-250. doi: 10.1002/sm2.77

18. Pakpour AH, Yekaninejad MS, Nikoobakht MR, Burri A, Fridlund B. Psychometric properties of the Iranian version of the premature ejaculation diagnostic tool. Sex Med. 2014;2(1):31-40.

19. Althof SE, Psychosexual therapy for premature ejaculation. Transl Androl Urol. 2016;5(4):475-481. doi: 10.21037/ tau.2016.05.15.12

20. Hashmi AM, Naz S, Asif A, Khawaja IS. Urdu translation of the Hamilton Rating Scale for Depression: Results of a validation study. Pak J Med Sci. 2016;32(6):1479. doi: $10.12669 /$ pjms.326.11399

\section{Authors Contribution:}

MUB: Did Literature search, data analysis, data interpretation, write-up, and is responsible and accountable for the accuracy or integrity of the work.

WA: Conceptualized the study design, data analysis, and data interpretation.

MS: Did Data Collection and write-up.

MHA: Did Urdu Translation. 